\title{
Etude expérimentale et modélisation d'un stockage thermique de longue durée en lit de cailloux enterré, couplé à des capteurs solaires à air
}

\author{
C. Dang Vu $(*)$ et B. Delcambre $\left(^{+}\right)$ \\ (*) Equipe RAMSES (E.R. 293 du CNRS), Bât. 508, Centre Universitaire, 91405 Orsay, France \\ $\left(^{+}\right)$C.S.T.B., route des Lucioles, Sophia Antipolis, B.P. 21, 06561 Valbonne Cedex, France
}

(Reçu le 31 octobre 1986, accepté le 9 mars 1987)

\begin{abstract}
Résumé. - Nous présentons l'analyse d'ensemble d'une expérimentation de stockage thermique de longue durée en lit de cailloux, réalisée à l'Etablissement C.S.T.B. de Sophia Antipolis (Valbonne) en collaboration avec le CNRS (Orsay). Le réservoir de cailloux de $160 \mathrm{~m}^{3}$, enterré et isolé, était ventilé soit par une boucle aéraulique avec préchauffage éventuel de l'air soufflé, soit par de l'air extrait d'un champ de capteurs solaires à air. Ce montage a permis tout un jeu d'essais de charge et décharge thermiques, entrecoupés de périodes de relaxation. Les relevés thermiques durant les périodes de relaxation ont fait l'objet d'une analyse numérique qui a mis en évidence les pertes vers le sol et les effets de convection naturelle au sein du stockage. Cette expérimentation permet ainsi de mieux cerner les limites d'efficacité d'un stockage de longue durée (quelques semaines) en lits de roches de grand volume.
\end{abstract}

\begin{abstract}
We present the overall results and analysis of a long term rock-bed thermal storage experiment carried out at the C.S.T.B. Centre of Sophia Antipolis (Valbonne), in collaboration with CNRS (Orsay). The pebbles tank $\left(160 \mathrm{~m}^{3}\right)$ was excavated directly in the earth and insulated; there were two means to blow and extract air in/from the pebbles tank : either through an air loop where air could be preheated, or from a field of air solar collectors. This installation allowed various sets of thermal charges and discharges, separated by relaxation periods. The thermal measurements during these relaxation periods were analysed by comparison with a numerical model ; this analysis exhibit clearly the thermal losses of the storage system towards the surrounding soil, reinforced by spontaneous convection arising within the pebble medium. This experiment allows one to precisely define the limits of efficiency of large rock-bed storage systems over a time period of several weeks.
\end{abstract}

\section{Nomenclature.}

$C_{\mathrm{c}}$ : chaleur massique des cailloux $\left(\mathrm{J} / \mathrm{kg}{ }^{\circ} \mathrm{C}\right)$

$C_{\mathrm{f}}$ : chaleur massique du fluide caloporteur $\left(\mathrm{J} / \mathrm{kg}^{\circ} \mathrm{C}\right)$

$C_{\mathrm{s}}:$ chaleur massique du sol $\left(\mathrm{J} / \mathrm{kg}{ }^{\circ} \mathrm{C}\right)$

$d_{\mathrm{c}}$ : diamètre moyen des cailloux $(\mathrm{m})$

$d_{\text {sol }}$ : diamètre moyen des éléments de matériau divisé (dont cailloux) utilisés dans les diverses expériences de mesure de transfert thermique en milieu divisé (Tableau I)

g : vecteur accélération de la pesanteur $\left(\mathrm{m} / \mathrm{s}^{2}\right)$

$G$ : débit massique du fluide par unité d'aire dans le stockage supposé vide $\left(\mathrm{kg} / \mathrm{s} \mathrm{m}^{2}\right) G=$ $\varepsilon \rho v=\rho v_{0}$

$H \quad$ : hauteur du lit de cailloux (m) $h$ : coefficient d'échange thermique surfacique fluide-cailloux $\left(\mathrm{W} / \mathrm{m}^{2}{ }^{\circ} \mathrm{C}\right)$

$h_{\mathrm{v}}$ : coefficient d'échange thermique volumique fluide-cailloux $\left(\mathrm{W} / \mathrm{m}^{3}{ }^{\circ} \mathrm{C}\right)$

$h_{\mathrm{p}}$ : coefficient d'échange thermique de la paroi de l'enceinte $\left(\mathrm{W} / \mathrm{m}^{2}{ }^{\circ} \mathrm{C}\right)$

$K$ : perméabilité du milieu air/cailloux $\left(\mathrm{m}^{2}\right)$

$L \quad:$ longueur du stockage $(\mathrm{m})$

$p$ : pression moyenne locale $\left(\mathrm{kg} / \mathrm{m} \mathrm{s}^{2}\right)$

$\Delta p \quad$ : perte de charge dans le stockage $\left(\mathrm{kg} / \mathrm{m} \mathrm{s}^{2}\right)$

$S \quad$ : surface des cailloux par unité de volume du milieu composite $\left(\mathrm{m}^{2} / \mathrm{m}^{3}\right)$

$T_{\mathrm{B}}:$ température du béton $\left({ }^{\circ} \mathrm{C}\right)$

$T_{\mathrm{c}} \quad:$ température moyenne locale des cailloux $\left({ }^{\circ} \mathrm{C}\right)$

$T^{*}$ : température locale équivalente du milieu composite air/cailloux $\left({ }^{\circ} \mathrm{C}\right)$ 
$T_{\mathrm{ex}}:$ température de l'air extérieur $\left({ }^{\circ} \mathrm{C}\right)$

$T_{\mathrm{f}}$ : température moyenne locale du fluide caloporteur $\left({ }^{\circ} \mathrm{C}\right)$

$T_{\mathrm{s}} \quad$ : température du sol $\left({ }^{\circ} \mathrm{C}\right)$

$T_{0} \quad$ : température de référence du fluide $\left({ }^{\circ} \mathrm{C}\right)$

$\mathbf{v}$ : vitesse interstitielle du fluide caloporteur $(\mathrm{m} / \mathrm{s})$

$\mathbf{v}_{0} \quad$ : vitesse de filtration

$V$ : volume du stockage $\left(\mathrm{m}^{3}\right)$

$\beta$ : coefficient d'expansion thermique du fluide $\left({ }^{\circ} \mathrm{C}^{-1}\right)$

$\varepsilon \quad:$ porosité du milieu air/cailloux

$\lambda_{c}:$ conductivité thermique des cailloux $\left(\mathrm{W} / \mathrm{m}{ }^{\circ} \mathrm{C}\right)$

$\lambda^{*}$ : conductivité thermique équivalente du milieu composite air/cailloux $\left(\mathrm{W} / \mathrm{m}{ }^{\circ} \mathrm{C}\right)$

$\lambda_{\mathrm{f}}$ : conductivité thermique du fluide $\left(\mathrm{W} / \mathrm{m}{ }^{\circ} \mathrm{C}\right)$

$\lambda_{\mathrm{s}}$ : conductivité thermique du sol $\left(\mathrm{W} / \mathrm{m}^{\circ} \mathrm{C}\right)$

\section{Introduction.}

Le stockage de longue durée de l'énergie dans de grandes masses de matériaux naturels (grands réservoirs d'eau, ou sol) est l'objet d'études expérimentales et théoriques nombreuses depuis quelques années [30]. Les conférences spécialisées, ENERSTOCK83 et ENERSTOCK-85 [28, 29], ont permis récemment de faire le point sur ces travaux. Nous nous sommes attachés, depuis une première étude de dimensionnement [8], à explorer par l'expérimentation et la modélisation une solution de stockage thermique en matériau naturel aisée à mettre en œuvre : les lits de cailloux ou galets ventilés par de l'air pulsé (chaud ou froid). Cet article présente une synthèse des résultats obtenus depuis cinq ans.

Le stockage thermique en lit de cailloux est assez couramment utilisé aux Etats-Unis ou en Australie dans l'habitat solaire [31]. Ce sont des stockages de courte durée (jour/nuit). Cette technique de stockage nécessite - à capacité égale - des volumes environ trois fois plus importants que les stockages en réservoirs d'eau; mais elle offre, en revanche, des possibilités techniques intéressantes :

- ces stockages peuvent être facilement associés à un champ de capteurs solaires à air, très efficaces grâce à leur faible inertie thermique ; ce système solaire peut être facilement couplé à un chauffage par air pulsé [31] ;

- cette technique est aisée à mettre en œuvre ; elle présente une insensibilité au gel et des risques limités de corrosion;

- enfin, la stratification thermique du stockage $[5,26]$ devrait permettre une restitution de la chaleur à un niveau élevé de température.

Dans une première étape de notre travail [9], nous avons réalisé la simulation, pour deux années consécutives de données météorologiques du site de $\nu$ : viscosité cinématique du fluide $\left(\mathrm{m}^{2} / \mathrm{s}\right)$

$\rho_{\mathrm{c}}:$ masse volumique locale moyenne des cailloux $\left(\mathrm{kg} / \mathrm{m}^{3}\right)$

$\rho_{\mathrm{f}}$ : masse volumique locale moyenne du fluide caloporteur $\left(\mathrm{kg} / \mathrm{m}^{3}\right)$

$\rho_{\mathrm{s}}:$ masse volumique locale du sol $\left(\mathrm{kg} / \mathrm{m}^{3}\right)$

$\rho_{0}$ : masse volumique du fluide à la température de référence $\left(\mathrm{kg} / \mathrm{m}^{3}\right)$

$N u=\frac{h d_{\text {sol }}}{\lambda_{\mathrm{f}}}$

$\operatorname{Pr}=\frac{\rho_{\mathrm{f}} C_{\mathrm{f}}}{\lambda_{\mathrm{f}}}:$ nombre de Prandtl

$R_{\mathrm{a}}^{*}=\frac{\beta(\rho C)_{\mathrm{f}}}{\nu} \frac{K}{\lambda^{*}} g \Delta T H:$ nombre de Rayleigh

$\operatorname{Re}=\frac{v_{0} d_{\text {sol }}}{\nu}:$ nombre de Reynolds du milieu divisé

Trappes, d'un système de stockage intersaisonnier comprenant un stockage cylindrique rempli de galets (volume $\left.=850 \mathrm{~m}^{3}\right)$, un champ de capteurs à air pouvant varier de 120 à $180 \mathrm{~m}^{2}$, et trois pavillons de $100 \mathrm{~m}^{2}$ environ (soit $900 \mathrm{~m}^{3}$ à chauffer). Cette simulation tenait compte uniquement des équations gouvernant la charge et la décharge des calories quand le stockage est ventilé, et des pertes vers le sol par le biais d'une conduction à symétrie cylindrique dans la masse air/cailloux et dans le sol supposé homogène.

De 1979 à 1981, l'équipe RAMSES avait réalisé le suivi détaillé d'une maison solaire en région parisienne (Dourdan), équipée d'un champ de capteurs à air de $70 \mathrm{~m}^{2}$ et d'un stockage en réservoir de cailloux de $40 \mathrm{~m}^{3}$. Les données recueillies ont pu être comparées aux prédictions d'une simulation numérique [5]. Les relevés détaillés effectués dans la masse du stockage (carte des températures) ont fait apparaître des effets divergeant fortement des hypothèses simplifiées fondant la simulation (zones du stockage non actives, chemins préférentiels de ventilation, pertes très dissymétriques sur les faces du stockage); de plus, ces effets pénalisaient très fortement le rendement du stockage. Il semblait donc délicat - sur la base de ces observations d'extrapoler au cas d'un stockage de longue durée, une simulation trop simplifiée.

C'est ainsi que fut entreprise en 1981, à l'Etablissement C.S.T.B. de Sophia Antipolis, une expérimentation de stockage thermique dans un réservoir parallélépipédique horizontal, enterré, contenant $160 \mathrm{~m}^{3}$ de cailloux. Le but de cette expérience était notamment de tester la possibilité d'un stockage de longue durée dans un lit de cailloux enterré. La configuration et le dispositif métrologique s'inspiraient de l'expérience mentionnée ci-dessus [5]. Des résultats partiels de cette expérience ont déjà été présentés $[3,3$ bis, 10]. Nous allons résumer l'analyse 
Tableau I. - Evaluations des coefficients de transfert thermique air/milieu divisé volumique, $h_{v}$, et surfacique, $h_{\mathrm{s}}$, obtenues à partir de douze références, rangées par ordre chronologique (de 1930 à 1982). Les valeurs indiquées dans les colonnes de droite correspondent au cas de référence (caractéristiques du matériau) indiqué dans le texte (\$2.2).

[Evaluations of the air/broken solids heat transfert coefficients, volumic, $h_{\mathrm{v}}$, and surfacic, $h_{\mathrm{s}}$, obtained from twelve chronologically-ordered references (from 1930 to 1982). The values in the columns, from left to right, correspond to case referred to in the text (material characteristics) (\$2.2).]

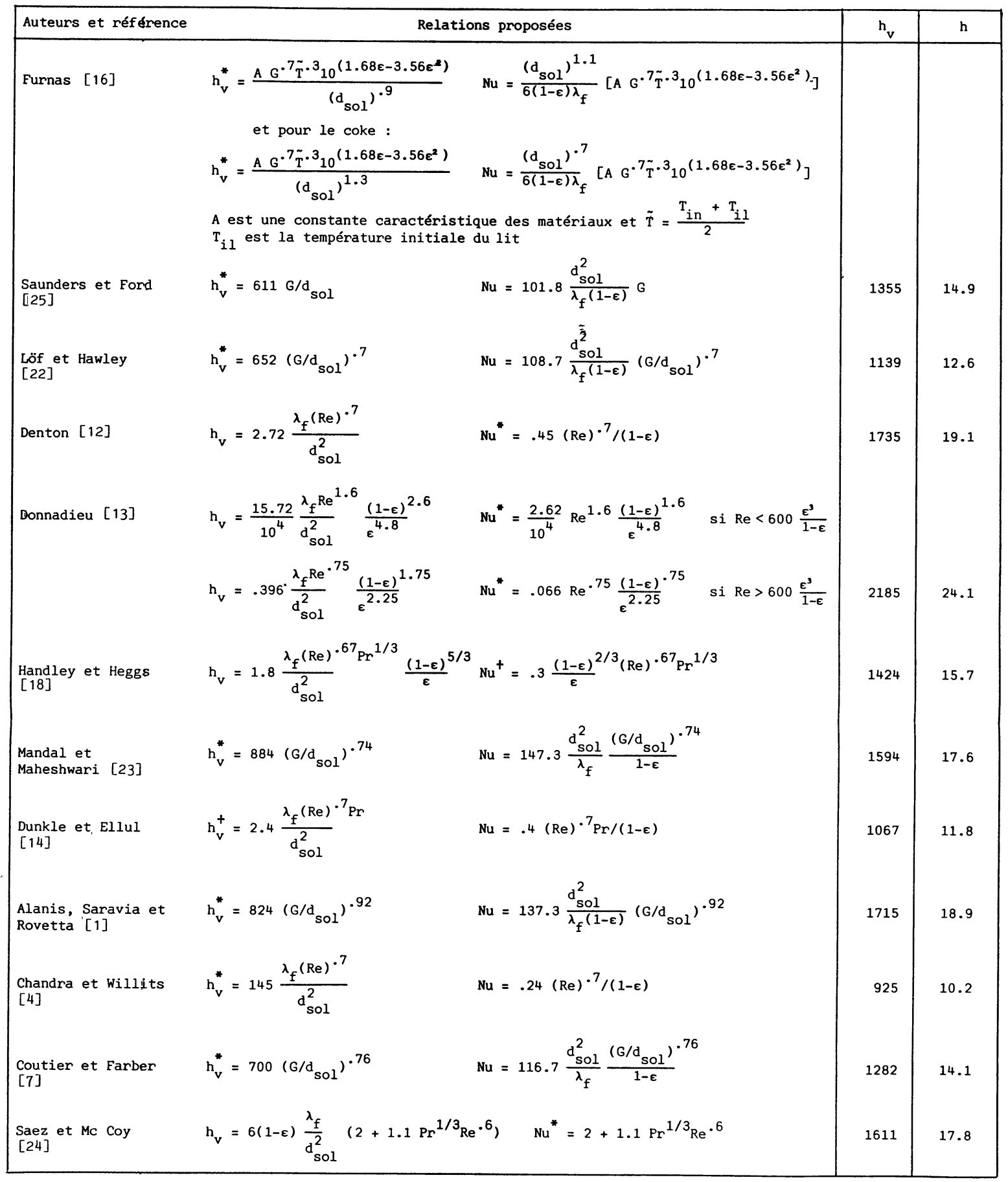

* Formule donnée dans la référence ; + formule dérivée de la référence.

complète des données recueillies pendant cette longue expérimentation qui a par ailleurs fait l'objet d'une thèse de doctorat [11].

Nous rappelons d'abord la mise en équations du problème, et discutons l'un des coefficients de transfert mis en jeu. Puis nous présentons le dispositif expérimental, et un échantillon de données recueillies. Celles-ci sont comparées, au paragraphe 4 , à un modèle de simulation détaillée. 


\section{Bases du modèle de stockage.}

Le stockage thermique que nous souhaitons modéliser peut être représenté (Fig. 1) comme un cylindre horizontal enterré, rempli de cailloux, où l'on peut souffler mécaniquement de l'air chaud dans une direction, de l'air froid dans l'autre, ce qui permet ainsi la charge ou la décharge thermique du stockage. Nous présentons d'abord la mise en équations du problème, puis l'un des paramètres physiques mis en jeu, le coefficient d'échange thermique surfacique air/cailloux, $h$.

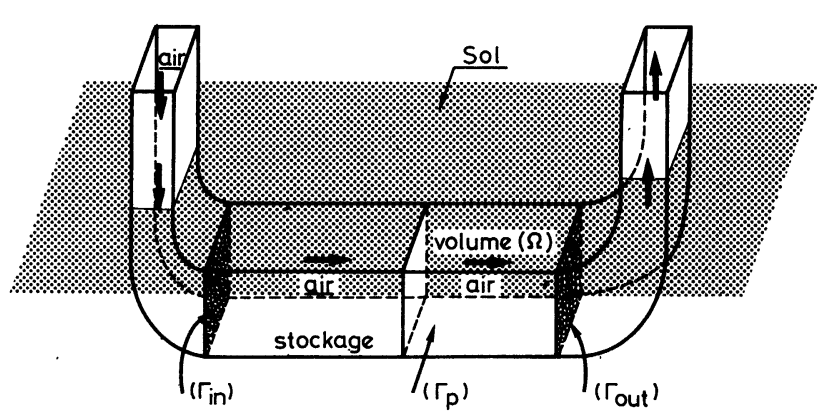

Fig. 1. - Schéma du stockage thermique en lit de cailloux enterré.

[Schematic diagram of the underground rock-bed thermal storage.]

2.1 MiSE EN ÉQUATIONS. - Les équations régissant le phénomène de stockage/déstockage de la chaleur dans le milieu composite air/cailloux s'écrivent :

Propagation de la chaleur dans le fluide:

$$
\begin{aligned}
\varepsilon\left[C_{\mathrm{f}} \rho_{\mathrm{f}}\left(\frac{\partial T_{\mathrm{f}}}{\partial t}+\mathbf{v} \cdot \nabla T_{\mathrm{f}}\right)\right. & \left.-\lambda_{\mathrm{f}} \Delta T_{\mathrm{f}}\right]= \\
= & h(\mathrm{v}) \cdot S \cdot\left(T_{\mathrm{c}}-T_{\mathrm{f}}\right) .
\end{aligned}
$$

Propagation de la chaleur dans les cailloux :

$$
\begin{aligned}
&(1-\varepsilon)\left[C_{\mathrm{c}} \rho_{\mathrm{c}} \frac{\partial T_{\mathrm{c}}}{\partial t}-\lambda_{\mathrm{c}} \Delta T_{\mathrm{c}}\right]= \\
&=-h(\mathrm{v}) . S .\left(T_{\mathrm{c}}-T_{\mathrm{f}}\right) .
\end{aligned}
$$

Conservation de la masse fluide :

$$
\varepsilon \frac{\partial \rho_{\mathrm{f}}}{\partial t}+\operatorname{div}\left(\varepsilon \rho_{\mathrm{f}} \mathbf{v}\right)=0
$$

$\underline{\text { Equation d'état : }}$

$$
\rho_{\mathrm{f}}=\rho_{0}\left(1-\beta \cdot\left(T-T_{0}\right)\right) .
$$

Loi de Darcy :

$$
\rho_{\mathrm{f}} \frac{\partial \mathbf{v}}{\partial t}=-\nabla p+\rho_{\mathrm{f}} \mathbf{g}-\frac{\rho_{\mathrm{f}} v \varepsilon \mathbf{v}}{K} .
$$

Les conditions initiales et aux limites sont les suivantes :

Conditions initiales :

$$
\begin{aligned}
T_{\mathrm{f}}(x, 0) & =T_{\mathrm{f}}^{0}(x) \\
T_{\mathrm{c}}(x, 0) & =T_{\mathrm{c}}^{0}(x) \\
p(x, 0) & =p^{0}(x)
\end{aligned}
$$

où $x \in \Omega$, volume intérieur à l'enceinte.

Conditions aux limites dans le cas d'un réservoir enterré :

- Conditions sur $\Gamma_{\mathrm{p}}$, surfaces des parois de l'enceinte :

$$
\begin{aligned}
-\lambda_{\mathrm{f}} \frac{\partial T_{\mathrm{f}}}{\partial \mathbf{n}} & =h_{\mathrm{p}} \cdot\left(T_{\mathrm{f}}-T_{\mathrm{s}}\right) \\
-\lambda_{\mathrm{c}} \frac{\partial T_{\mathrm{c}}}{\partial \mathbf{n}} & =h_{\mathrm{p}} \cdot\left(T_{\mathrm{c}}-T_{\mathrm{s}}\right) \\
\mathbf{v} \cdot \mathbf{n} & =0 .
\end{aligned}
$$

Nous marquerons désormais par l'indice « in » les grandeurs physiques à l'entrée du lit, et par « out » celles à la sortie.

- Conditions sur $\Gamma_{\text {in }}$, surface d'entrée du fluide caloporteur

$$
\begin{aligned}
T_{\mathrm{f}} & =T_{\text {in }} \\
-\lambda_{\mathrm{c}} \frac{\partial T_{\mathrm{c}}}{\partial \mathbf{n}} & =h \cdot\left(T_{\mathrm{c}}-T_{\mathrm{f}}\right) \\
p & =p_{\text {in }} .
\end{aligned}
$$

- Conditions sur $\Gamma_{\text {out }}$, surface de sortie du fluide :

$$
\begin{aligned}
\frac{\partial T_{\mathrm{f}}}{\partial \mathbf{n}} & =0 \\
-\lambda_{\mathrm{c}} \frac{\partial T_{\mathrm{c}}}{\partial \mathbf{n}} & =h \cdot\left(T_{\mathrm{c}}-T_{\mathrm{f}}\right) \\
p & =p_{\text {out }} .
\end{aligned}
$$

Dans (11), (13), (14), $\mathbf{n}$ est le champ de normales à la surface de l'enceinte.

Les quantités $T_{\mathrm{f}}(x, t), T_{\mathrm{c}}(x, t), p(x, t), \rho_{\mathrm{f}}(x, t)$ sont entendues comme des moyennes locales, au voisinage de $x$, caractérisant le milieu composite fluide/cailloux. La vitesse moyenne du fluide $\mathbf{v}$ dans les interstices est appelée « vitesse intersticielle »; la vitesse théorique en l'absence de substrat solide est appelée «vitesse de filtration» $\mathbf{v}_{0}: \mathbf{v}_{0}=\varepsilon \mathbf{v}$. En première approximation $C_{\mathrm{f}}, C_{\mathrm{c}}, \lambda_{\mathrm{f}}, \lambda_{\mathrm{c}}, \rho_{\mathrm{c}}, \nu$, sont des quantités qui peuvent être considérées comme constantes. Par ailleurs, $\varepsilon, S, K$, sont des paramètres qui caractérisent la granulométrie et la configuration locale des cailloux ; ils sont éventuellement fonctions de $x$ mais non du temps. Enfin $h(v)$ désigne le coefficient de transfert thermique fluide-cailloux. Ainsi les seconds membres de (1) et (2) dépendent 
éventuellement de $\mathbf{v}$ via cette fonction. En première approximation $\varepsilon, S, K$ et $h(v)=h$, peuvent être considérés comme des constantes. Les conditions aux limites sont standards, sauf peut-être (11) et (14) qui supposent que le coefficient moyen d'échange fluide-cailloux est le même sur les surfaces d'entrée et de sortie qu'à l'intérieur du milieu alors que le régime d'écoulement $\mathrm{y}$ est différent.

\subsection{COEFFICIENT DE TRANSFERT THERMIQUE} AIR/CAILlOUX, $h .-$ Dans la mise en équations précédente, il apparaît un paramètre assez sensible pour la dynamique du stockage/déstockage, le coefficient de transfert thermique air/cailloux, $h$, qui conditionne notamment la forme du front thermique dans le stockage.

Ce paramètre a fait l'objet de nombreuses études et évaluations depuis l'article original de Schumann [26]. Dans le tableau I, nous avons regroupé des évaluations recueillies dans douze articles anciens et récents, sur divers matériaux divisés (billes de verre, d'alumine, cailloux, etc.). Pour chaque référence nous donnons la formulation adoptée pour $h_{\mathrm{v}}$ et $\mathrm{Nu}$ ainsi que les valeurs obtenues pour $h_{\mathrm{v}}$, coefficient volumique, et $h$, coefficient surfacique ; ces valeurs sont estimées à partir des données de chaque article en se ramenant au même type de matériau de référence, caractérisé par :

$d_{\text {sol }}$ (diamètre moyen des éléments de solide)

$|\mathbf{v}|$ (vitesse moyenne du fluide) $=0,1 \mathrm{~m} / \mathrm{s}$ $=4,5 \mathrm{~cm}$

$\varepsilon$ (porosité du milieu composite) $=0,32$

$\operatorname{Pr}(\mathrm{Nb}$. de Prandtl pour l'air) $=0,7$

Remarquons que la relation liant $h$ et $h_{\mathrm{v}}$ :

$$
h=\left[d_{\text {sol }} /(6(1-\varepsilon))\right] h_{\mathrm{v}}
$$

n'est réellement valable que pour un lit de sphères.

Le tableau I montre une variation de la valeur de $h$, pour le cas de référence, allant du simple au double suivant la formulation choisie. Dans la référence [11], nous avons analysé les différentes formulations à la fois sur le plan des hypothèses des modèles et des conditions d'expérimentation : plages de variation des diamètres $d_{\text {sol }}$, de la porosité $\varepsilon$, du type de matériau utilisé, du débit massique ou nombre de Reynolds du fluide. Nous avons mis en évidence la grande dépendance des coefficients numériques des diverses relations, par rapport à ces conditions expérimentales.

Toutes ces relations donnent un paramètre caractéristique d'un échange local en fonction de grandeurs moyennes, donc globales. Ces grandeurs globales mesurées masquent les phénomènes locaux qui peuvent être importants ; les nombres de Reynolds rencontrés sont du domaine du régime laminaire; Handley et Heggs [18] ont fait remarquer qu'aucune mesure ne permettait d'affirmer l'existence d'un flux laminaire bien établi : les discontinuités du lit peuvent donner naissance à des turbulences dans la couche limite laminaire qui ont sans doute un impact non négligeable sur le coefficient $h$. Handley et Heggs donnent une relation empirique entre $h$ et la perte de charge dans le lit avec des coefficients dépendant du nombre de bifurcations dans le fluide.

Néanmoins, les différentes simulations du stockage/déstockage ont montré que pour notre problème l'évolution du stockage était peu sensible à ces incertitudes sur la valeur du coefficient $h$.

\section{Dispositif expérimental de stockage en lit de cailloux enterré.}

Le prototype expérimental de stockage en lit de cailloux enterré a été construit au Centre C.S.T.B. de Sophia Antipolis, de fin 80 à début 82 . Il s'agissait à la fois d'expérimenter cette filière de stockage du point de vue technique et économique, mais aussi de pouvoir mener une expérimentation sur le stockage lui-même. Dans ce but, on a prévu deux types de couplage aéraulique du stockage: soit avec un champ de capteurs à air dans un but de démonstration, soit avec une boucle aéraulique modulable en débit et température dans un but d'expérimentation.

Le volume de cailloux enterré est de $160 \mathrm{~m}^{3}$ (longueur : $20 \mathrm{~m}$; largeur: $3,2 \mathrm{~m}$; hauteur : $2,5 \mathrm{~m})$; la cuve de stockage est flanquée d'une galerie technique par où l'on peut accéder notamment aux diverses mesures physiques et aux plenums de soufflage ou d'extraction d'air (Fig. 2). L'ensemble du dispositif est décrit en détail dans les références [3] et [11].

La longueur du stockage et le diamètre moyen des cailloux $(5 \mathrm{~cm})$ ont été optimisés, d'une part pour limiter les pertes de charge et obtenir un bon échange thermique air/cailloux, d'autre part pour permettre une charge ou une décharge complète (retour à l'état initial) dans un temps relativement court, ce stockage ayant été construit dans un but d'expérimentations.

Deux points techniques sensibles sont apparus lors de la construction et de l'expérimentation : l'étanchéité aéraulique de l'unité de stockage et des gaines de circulation d'air et le calibrage des cailloux [3]. Les mesures de débit d'air dans le stockage ont montré que les infiltrations ou les fuites d'air représentent 8 à $12 \%$ du débit total. Le remplissage du stockage a été fait avec des galets préalablement lavés, sélectionnés par passage à travers deux cribles de diamètre $3,5 \mathrm{~cm}$ et $6,5 \mathrm{~cm}$.

La capacité thermique totale du stockage (interne à l'enceinte isolante) est délicate à déterminer avec précision, en raison des difficultés pour apprécier certains paramètres : porosité $(\simeq 0,35)$; capacités thermiques des galets et du béton. Notre évaluation de cette capacité totale est : 72 à $85 \mathrm{kWh} /{ }^{\circ} \mathrm{C}$. 


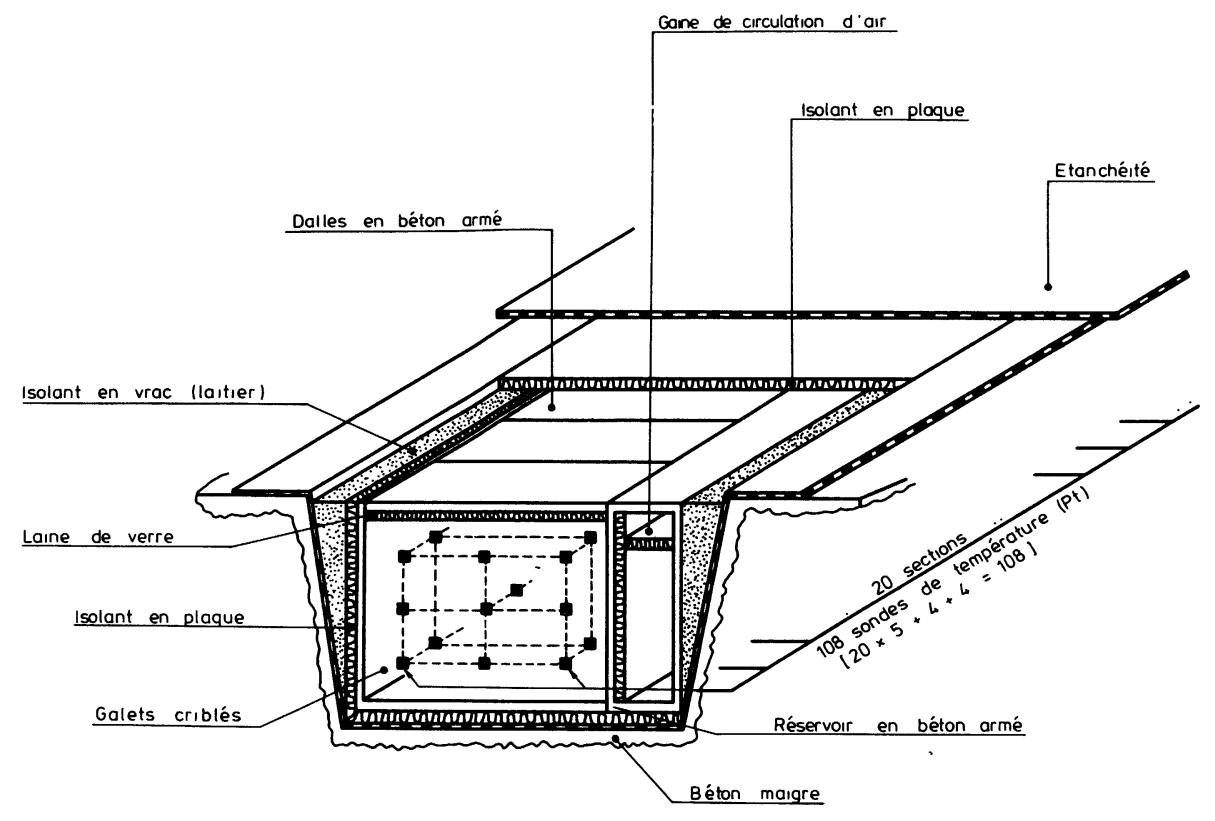

Fig. 2. - Coupe schématique du réservoir enterré de $160 \mathrm{~m}^{3}$ de cailloux de l'Etablissèment C.S.T.B. de Sophia Antipolis ayant permis l'expérimentation de stockage thermique. L'emplacement des sondes thermiques dans la masse caillouteuse est indiqué ( 18 sections avec 5 relevés de température, 2 sections aux extrémités avec 9 relevés de température ; total $=108$ sondes $)$.

[Schematic section of the $160 \mathrm{~m}^{3}$ underground pebbles tank, constructed at the C.S.T.B. Centre at Sophia Antipolis (South of France). The thermal probes in the pebbles medium are indicated ( 18 sections with 5 thermal probes, 2 endsections with 9 thermal probes ; total $=108$ probes).]

\section{Présentation des données recueillies en cours d'expérience.}

De début 82 à l'automne 83 se sont succédé une vingtaine de cycles d'expérimentation thermique où le stockage était couplé à la boucle aéraulique. Après une période de séchage du stockage, nous avons programmé divers cycles de charge ou décharge du stockage en faisant varier les valeurs de débit ou de température de l'air soufflé (ou extrait) ou en modifiant les conditions de soufflage d'air (masques à l'entrée du stockage), les valeurs nominales de débit et température restant fixes au cours de ces essais. De même, nous avons observé l'évolution du stockage au cours de divers cycles de relaxation thermique. Au-delà de ces essais simples ont été programmés des essais plus complexes associant par exemple une période longue de charges thermiques diurnes puis une décharge intermittente après relaxation, c'est-à-dire un cycle proche de ce que serait une opération de stockage-déstockage de longue durée, dans un système de chauffage solaire (débits et températures d'air variables). On trouvera en référence [11], p. 107 à 109, une présentation détaillée du plan d'expérience. Voyons maintenant quelques résultats importants extraits de ces nombreuses mesures.

\subsection{DÉTERMinATION DES PERTES DE CHARGE DANS} LE STOCKAGE. - Les points expérimentaux sont reportés sur la figure 3 . Les deux points donnés pour une même perte de charge correspondent au débit entrant et au débit sortant. La courbe en trait plein

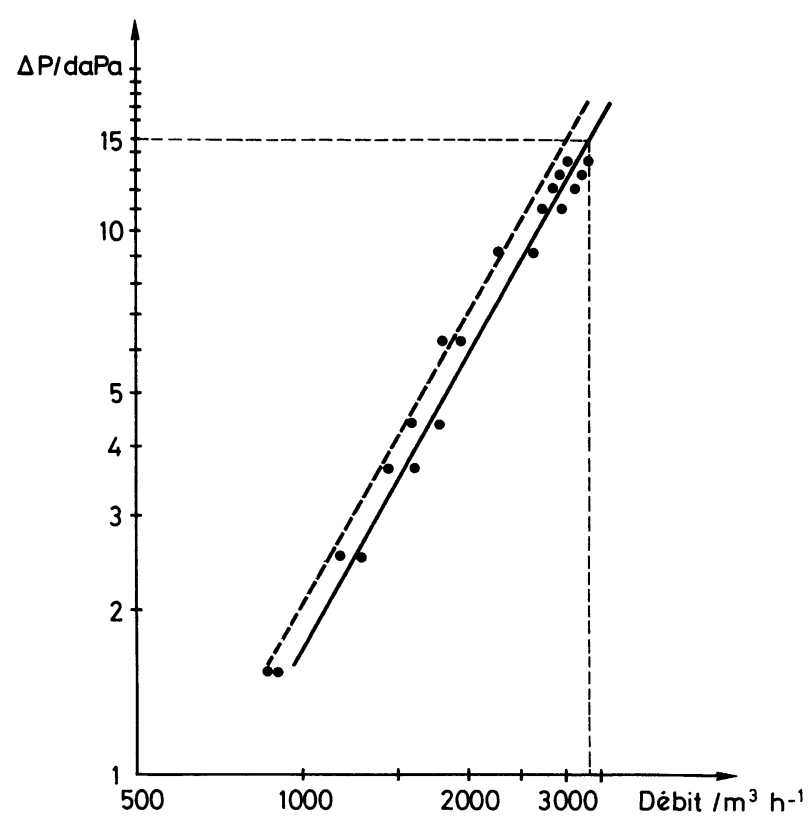

Fig. 3. - Pertes de charge dans le stockage en fonction du débit.

- trait continu : courbe expérimentale, trait pointillé : formule de Close [6].

[Pressure drop in the storage medium as a function of the flow rate.

- full line : experimental curve, dotted line : theoretical curve.] 
est la courbe déduite des points expérimentaux. La courbe en trait pointillé correspond à la formule de Close [6]. Rappelons que les cailloux utilisés dans l'expérience de Sophia Antipolis sont de granulométrie $35-65 \mathrm{~mm}$ et de formes variées. Il semble en résulter une perte de charge légèrement inférieure à la perte de charge d'un lit « arrangé au hasard » au sens de Dunkle et Ellul [14], alors qu'on aurait pu s'attendre, à cause de la taille non homogène des cailloux, à des pertes de charge plus importantes.

Une limitation à $150 \mathrm{~Pa}$ des pertes de charge dans le stockage a été déterminée par considération des autres pertes de charge (capteurs, gaine...) et de la consommation d'énergie électrique admissible des ventilateurs (10\% de l'énergie récupérée grâce au stockage).

4.2 EXPÉRIMENTATIONS DE CHARGE THERMIQUE. - Nous avons réalisé différentes expérimentations de charge thermique. Dans la mesure où l'expérimentation préalable [5] sur un stockage de $40 \mathrm{~m}^{3}$ avait montré de gros problèmes d'inhomogé- néité de répartition d'air en entrée ou sortie du stockage, nous avons également réalisé des essais sur ces problèmes de distribution d'air. A cet effet, des précautions particulières avaient été prises lors de la construction pour assurer la meilleure répartition possible du fluide à l'entrée et à la sortie du stockage. Cependant, ces grandes chambres de répartition représentent un coût supplémentaire ; aussi avons-nous voulu évaluer ce que serait l'impact d'une distribution inhomogène du fluide à l'entrée, sur la distribution des températures dans le stockage. Pour cela, nous avons fait plusieurs expériences de charge et de décharge où les $2 / 3$ des surfaces d'entrée et de sortie étaient obturées par des panneaux étanches, ou masques.

Le tableau II donne les caractéristiques de quatre essais de charge thermique : une sans masque, puis trois avec masques. Une dénomination abrégée (CHRA, etc.) marquée en quatrième colonne du tableau II permettra de repérer le type d'essai sur les graphiques donnant les résultats de ces essais (Fig. 4).

Tableau II. - Caractéristiques des divers essais de charge thermique du stockage.

[Characteristics of different tests of thermal charge of the storage.]

\begin{tabular}{|c|c|c|c|}
\hline $\begin{array}{l}\text { Caractéristiques } \\
\text { du masque } \\
\text { à l'entrée du fluide }\end{array}$ & $\begin{array}{c}\text { Débit d'air } \\
\mathrm{m}^{3} / \mathrm{h}\end{array}$ & $\begin{array}{l}\text { Figure } \\
\text { référencée }\end{array}$ & $\begin{array}{c}\text { Dénomination abrégée } \\
\text { de l'essai } \\
\text { sur les figures }\end{array}$ \\
\hline Pas de masques & 2530 & 4-A & $\frac{\text { CHRA }}{\text { (charge rapide) }}$ \\
\hline $\begin{array}{l}\text { Masques couvrant les } 2 / 3 \text { su- } \\
\text { périeurs des surfaces d'entrée } \\
\text { et de sortie }\end{array}$ & 2910 & 4-B & $\frac{\text { CHMASDTS }}{\text { (charge }} \frac{\text { rapide avec }}{\text { aux deux tiers supérieurs) }}$ \\
\hline $\begin{array}{l}\text { Masques couvrant les } 2 / 3 \text { infé- } \\
\text { rieurs des surfaces d'entrée et } \\
\text { de sortie }\end{array}$ & 2860 & $4-\mathrm{C}$ & $\begin{array}{l}\text { CHMASDTI } \\
\text { (charge } \\
\text { aux deux tiers inférieurs) }\end{array}$ \\
\hline $\begin{array}{l}\text { Masques couvrant les } 2 / 3 \text { su- } \\
\text { périeurs des surfaces d'entrée } \\
\text { et de sortie }\end{array}$ & 570 & 4-D & $\begin{array}{l}\text { (charge } \frac{\text { CHLMDTS }}{\text { lente avec }} \text { masques } \\
\text { aux deux tiers supérieurs) }\end{array}$ \\
\hline
\end{tabular}

Les trois graphiques (4A) montrent l'évolution du front chaud dans le stockage lors de l'expérience de Charge Rapide (CHRA). Chaque courbe est obtenue par lissage des points expérimentaux pour des relevés réalisés pendant une semaine à partir du début de l'essai de charge. Le front thermique entre les parties chaude et froide du stockage est net: l'échange entre l'air et les cailloux est donc de bonne qualité. La température est quasi homogène entre les différentes parties, le front chaud progressant un peu plus vite le long de l'axe central. On peut sans doute expliquer cet effet par le rôle non négligeable d'amortissement joué par l'enveloppe de béton. Le bas du stockage montre un léger retard à la charge.

L'expérience «CHMASDTS » consiste en une charge rapide avec masques appliqués sur les $2 / 3$ supérieurs des surfaces d'entrée et de sortie. Les graphiques (4B) montrent une évolution des températures dans le stockage très similaire à celle de la charge sans masques; nous notons seulement que dans ce cas, haut et bas du stockage sont à la même température en fin de charge. Les masques ont donc 

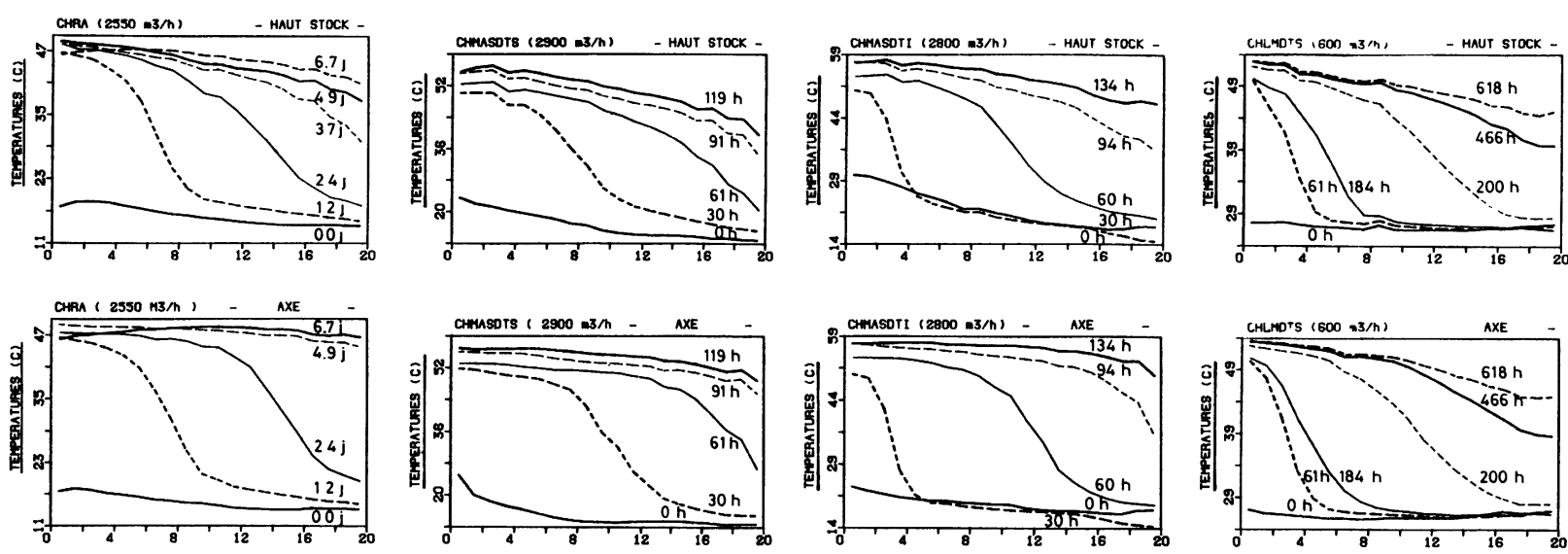

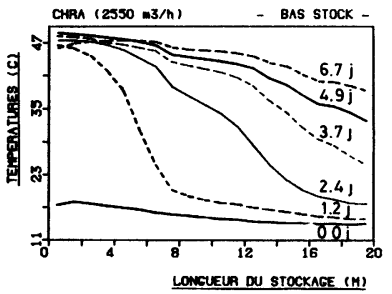

A

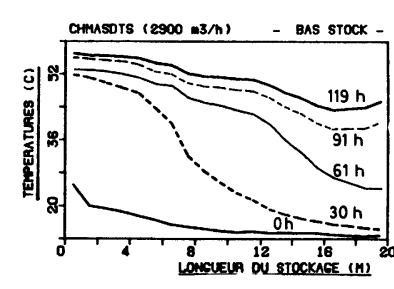

B

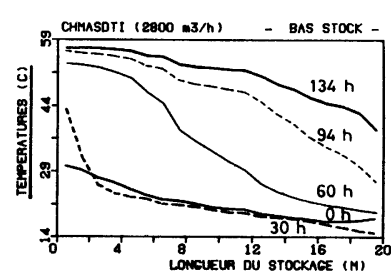

C

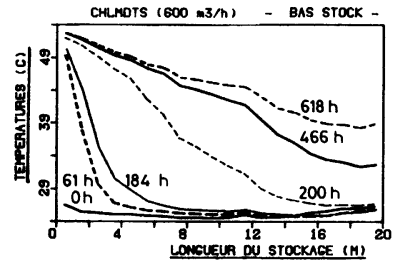

D

Fig. 4. - Evolution des températures le long du stockage pour les quatre cas d'essais de charge référencés au tableau II ; chaque cas (4A, 4B, 4C, 4D) est regroupé dans une colonne :

- figures du haut : température moyenne mesurée dans la zone haute du stockage ;

- figures du milieu : température sur l'axe du stockage ;

- figures du bas : température moyenne mesurée dans la zone basse du stockage.

La distance par rapport à l'entrée du stockage est donnée en abscisse. Les courbes (alternées en traits pleins et pointillés) indiquent les mesures faites à un ou plusieurs jours d'intervalle à partir de la mesure initiale, avant charge, à $t=0 \mathrm{~h}$; l'heure de relevé est indiquée sur chaque courbe.

[Temperature measurements along the flow direction as a function of time, for 4 charge tests referenced in table II ( $4 \mathrm{~A}$, 4B, 4C, 4D) ; one case by column :

- top diagramm : mean temperature measurements in the top zone ;

- middle diagram : temperatures measurements on axis ;

- bottom diagram : mean temperatures measurements in the bottom zone.

Temperatures are plotted against distance from the pebbles medium entry.

Each curve (dashed and full lines, alternatively) is separated from the following by one or more days : the hour of measurement is indicated on each curve.]

peu d'influence sur la distribution de l'air du stockage.

Dans l'expérience «CHMASDTI 》 (graphiques 4C), les masques obturaient les $2 / 3$ inférieurs des surfaces d'entrée et de sortie. Pendant les premières heures, on note une dissymétrie du front thermique entre le haut et le bas du réservoir. L'avancée du front est beaucoup moins rapide durant les premières heures de charge que dans l'expérience «CHMASDTS » ; ceci tient au fait que pendant les 24 premières heures de charge, c'est de l'air à $20^{\circ} \mathrm{C}$ qui a été soufflé à débit de $3550 \mathrm{~m}^{3} / \mathrm{h}$.

Dans les expériences précédentes, la convection forcée pouvait masquer d'éventuels effets de convection naturelle (convection mixte). Une expérience à débit plus faible $\left(570 \mathrm{~m}^{3} / \mathrm{h}\right)$ avec masques sur les $2 / 3$ supérieurs des caillebotis a donc été programmée
(CHLMDTS). Les graphiques (4D) montrent une nette asymétrie haut/bas du front qui ne peut s'expliquer entièrement par une moins bonne isolation de la dalle inférieure [11].

En résumant les effets des masques sur la répartition thermique dans le stockage, on peut signaler :

- un effet plus important à faible débit $\left(600 \mathrm{~m}^{3} / \mathrm{h}\right)$ qu'à débit élevé $\left(3000 \mathrm{~m}^{3} / \mathrm{h}\right)$; à faible débit, la charge thermique plus rapide en haut du stockage suggère un phénomène de convection naturelle ;

- un effet plus marqué en position basse qu'en position haute, l'air étant insufflé dans (ou aspiré hors) des plenums par le haut.

Dans tous les cas, les effets dus aux masques restent faibles sur le rendement du stockage.

\subsection{RÉSUlTATS DES EXPÉRIENCES DE RELAXATION}


THERMIQUE. - Plusieurs essais ont été réalisés pour suivre l'évolution des températures du stockage, pendant une période de repos, volets d'entrée et de sortie fermés. Un stockage de longue durée peut, en effet, ne pas être utilisé directement après une période de charge (cas d'intersaisons tempérés). Au départ, le stockage était soit uniformément chargé (stockage plein), soit présentait 2 zones à températures différentes (stockage partiellement plein).

Stockage initialement plein. - Le stockage ayant été amené à une température quasi uniforme de $45^{\circ} \mathrm{C}$, on l'a laissé en relaxation thermique, complètement isolé aérauliquement (volets entrée/sortie fermés), pendant deux mois. Au cours de cette période, les valeurs des sondes de température ont été enregistrées toutes les 2 heures. Nous avons analysé les données expérimentales du premier mois. Le tableau III, qui correspond aux graphiques (5A), donne les moyennes le long du stockage des températures relevées aux sondes, tous les cinq jours. Il montre des évolutions de températures différentes en haut, au milieu et en bas du stockage.

Tableau III. - Expérience de relaxation thermique à partir d'un stockage chargé à $45{ }^{\circ} \mathrm{C}$ environ (au $\left.9 / 4 / 82\right)$. Dans cinq colonnes, de gauche à droite, sont portées des valeurs moyennes de température calculées à partir de relevés effectués du 15/4 au $7 / 5$; pour chaque colonne on a également indiqué l'écart moyen, $\Delta \mathrm{T}$, avec les valeurs mesurées initialement (au 9/4). Les cinq valeurs de température, dans chaque colonne, sont les moyennes des mesures réalisées dans vingt sections le long du stockage (voir Fig. 2), respectivement en haut du stockage (HC, $\mathrm{HM})$, sur l'axe (M), et en bas du stockage (BC, BM).

[Experimentation on thermal relaxation of the storage preliminary heated $\left(45^{\circ} \mathrm{C}\right.$ approximately on day $\left.9 / 4 / 82\right)$. In the five columns from left to right are indicated mean values of storage temperatures, derived from measurements realized from the $15 / 4 / 82$ to the $7 / 5 / 82$; for each column the mean deviations, $\Delta T$, with initial values measured on the $9 / 4 / 82$, are indicated. The five values of temperature, in each column, are the averages of measurements collected in the twenty sections of the storage (cf. Fig. 2), respectively at the top of the storage (HC, $\mathrm{HM})$, on the axis (M), and at the bottom of the storage (BC, BM).]

\begin{tabular}{|l|c|c|c|c|c|c|c|c|c|c|c|}
\cline { 2 - 10 } \multicolumn{1}{c|}{} & $9 / 4$ & $15 / 4$ & $\Delta(15 / 4)$ & $22 / 4$ & $\Delta(22 / 4)$ & $27 / 4$ & $\Delta(27 / 4)$ & $2 / 5$ & $\Delta(2 / 5)$ & $7 / 5$ & $\Delta(7 / 5)$ \\
\hline HC & 45,4 & 43,6 & 1,8 & 40,7 & 4,7 & 38,6 & 6,8 & 36,8 & 8,6 & 35,2 & 10,2 \\
HM & 46,4 & 44,4 & 2,0 & 41,2 & 5,2 & 39,2 & 7,2 & 37,3 & 9,1 & 35,7 & 10,7 \\
M & 47,8 & 43,2 & 5,6 & 39,3 & 8,5 & 37,2 & 10,6 & 35,4 & 12,4 & 33,9 & 13,9 \\
BC & 43,9 & 38,4 & 5,5 & 35,2 & 8,7 & 33,4 & 10,4 & 31,9 & 12,0 & 30,6 & 13,3 \\
BM & 44,9 & 39,8 & 5,1 & 36,0 & 8,9 & 34,1 & 10,8 & 32,5 & 12,4 & 31,2 & 13,7 \\
\hline
\end{tabular}

Deux phénomènes peuvent concourir à cette dissymétrie haut bas :

a) la différence entre isolations des dalles inférieure $\left(h_{\mathrm{p}}=0,25 \mathrm{~W} / \mathrm{m}^{2} .{ }^{\circ} \mathrm{C}\right)$ et supérieure $\left(h_{\mathrm{p}}=\right.$ $\left.0,16 \mathrm{~W} / \mathrm{m}^{2} .{ }^{\circ} \mathrm{C}\right)$ du stockage ;

b) un courant de convection naturelle dans le stockage même où, initialement, la partie centrale est plus chaude.

En effet, la différence de température entre le haut et le milieu du stockage, $\Delta T$, est suffisamment faible devant la température de la zone médiane pour pouvoir utiliser l'hypothèse de Boussinesq. Dans ce cas, si on assimile le milieu air/cailloux à un milieu poreux, on peut se référer au critère d'appari- tion de mouvements convectifs dans une couche horizontale, mis en évidence par Horton, Roger et Lapwood [19, 21], à savoir : $R_{\mathrm{a}}^{*}>R_{\mathrm{ac}}^{*}=4 \pi^{2}$ (voir nomenclature). Pour un $\Delta T$ de l'ordre du degré, $R_{\mathrm{a}}^{*}$ est de l'ordre de 100 , supérieur au seuil ci-dessus $\left(4 \pi^{2}\right)$. On peut donc s'attendre, d'après ce calcul simple, à l'existence de courants convectifs dans le stockage.

Quant à l'homogénéisation au cours du temps, elle s'expliquerait en partie par le réchauffement du sol consécutif aux pertes du réservoir. Chaque phénomène ne peut cependant à lui seul expliquer les données expérimentales ; l'ensemble est à prendre en compte pour expliquer les résultats observés, qui sont regroupés sur la figure (5A). 


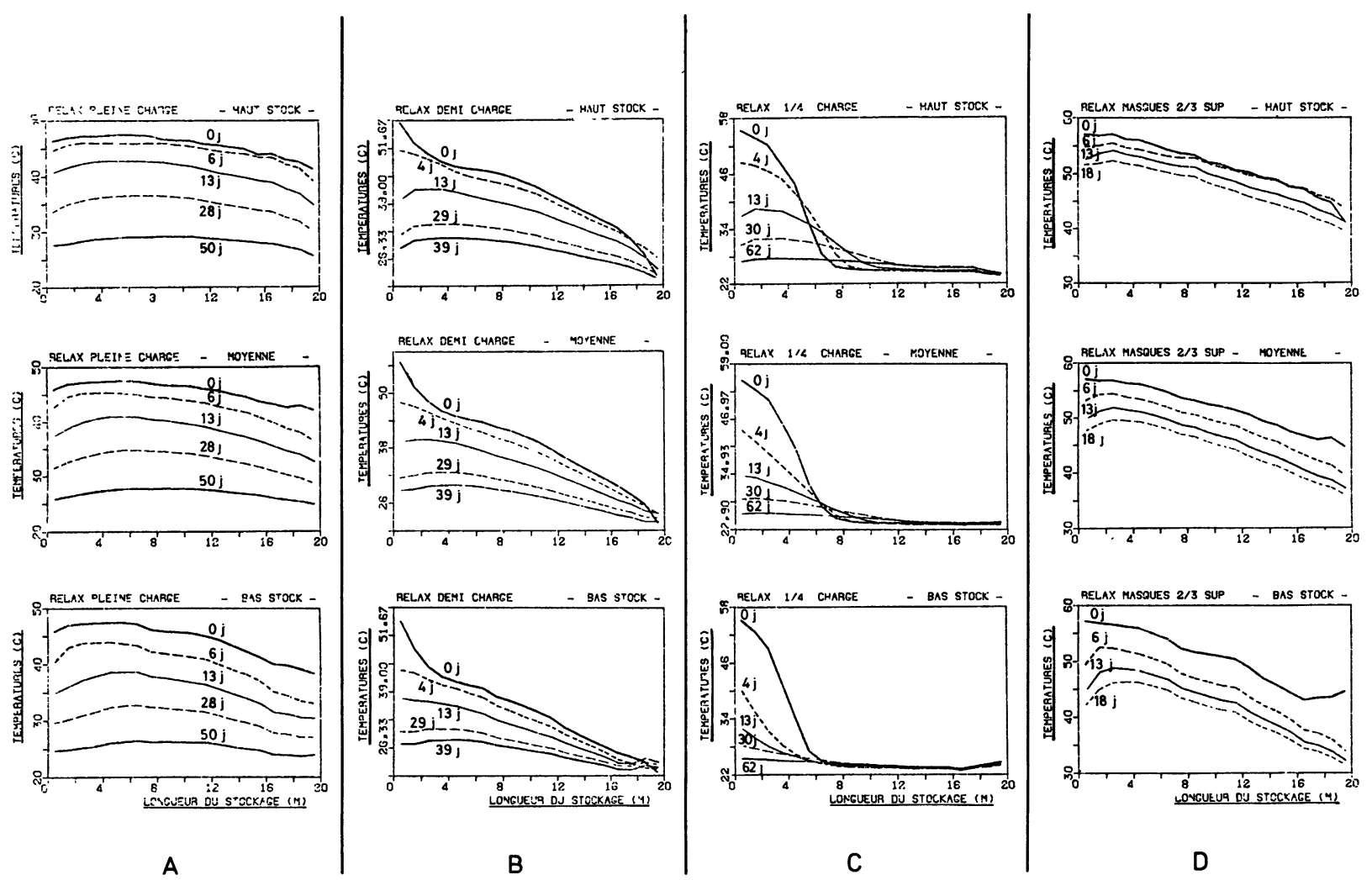

Fig. 5. - Relaxation thermique du stockage à partir de quatre états de charge thermique différents. Les courbes donnent la température moyenne en bas, au milieu et en haut du stockage, en fonction de la distance à l'entrée du stockage, et ce pour les cinq semaines suivant la charge thermique initiale. De gauche à droite, les quatre états de charge initiale du stockage correspondent respectivement à :

A : un stockage totalement chargé ;

B : un stockage chargé à moitié de sa capacité ;

C : un stockage chargé au quart de sa capacité ;

$\mathrm{D}$ : un stockage chargé aux deux tiers de sa capacité, avec des masques appliqués aux deux tiers supérieurs des surfaces d'entrée et de sortie.

[Thermal relaxation of the rock-bed storage system for 4 different levels of stored energy. Mean temperatures in the top, middle and bottom parts of the tank are plotted against distance from the storage entry surface, during the 5 weeks following the thermal charge period. From left to right, the 4 initial levels of stored energy :

A : thermal storage completely filled;

B : thermal storage half filled;

C : a quarter of the thermal storage is filled;

D : two thirds of the thermal storage is filled, with end-caps covering two third of the extreme sections.]

Stockage partiellement chargé. - Les figures (5B), (5C), (5D) donnent l'évolution des températures dans la masse caillouteuse en partant d'un stockage respectivement à demi, au quart et aux trois quarts chargé. Dans ce dernier cas, des masques hermétiques étaient appliqués sur les $2 / 3$ supérieurs des surfaces d'entrée et de sortie. On observe une dissymétrie dans l'évolution des températures entre le haut et le bas du stockage comme dans le cas du stockage initialement chargé (Fig. 5A) mais on note également un déplacement du front de chaleur vers la zone froide dans le haut du réservoir.

En conclusion, nous observons, pendant les périodes de relaxation, des pertes importantes dans les zones inférieure et médiane du réservoir, particulièrement les premiers jours, dues à une asymétrie haut/bas de l'isolation mais sans doute également à des courants de convection naturelle dans la masse du stockage.

\section{Modélisation du stockage.}

Dans une première étape de notre travail, précédant l'expérimentation de Sophia Antipolis, nous avions essayé diverses simulations de configurations théoriques du stockage en lit de cailloux. C'est ainsi que le système complet d'équations (1) à (15) avait fait l'objet d'une résolution numérique [17], par différen- 
ces finies à une dimension, pour le cas du déstockage. De plus, les deux équations thermiques avaient donné lieu à deux résolutions numériques, l'une approchée sur HP 97 [8], dans le cas d'un déstockage d'un volume cylindrique vertical isolé et enterré ; l'autre par différences finies, modélise l'évolution d'un stockage cylindrique horizontal, enterré, sans isolation, ainsi que celle du sol environnant, sans tenir compte d'éventuels effets convectifs [9].

5.1 CAS DU STOCKage EXPÉRIMENTÉ. - Dans les conditions concrètes du stockage de Sophia Antipolis, nous n'avons pu aborder la résolution numérique du modèle le plus général qui aurait pris en compte la conduction et la convection naturelle dans le stockage ainsi que la conduction dans le sol entourant le réservoir. Nous serions arrivés à un problème difficilement maniable avec nos moyens de calculs actuels.

Nous nous sommes limités à la modélisation numérique à trois dimensions de la conduction dans le milieu composite air/cailloux; les conditions aux limites tiennent compte des pertes à travers l'enveloppe considérées comme homogènes sur chaque face du stockage, et la température de la terre environnante est d'abord estimée par recalage du modèle sur les données expérimentales (nous n'avons pas de mesure directe dans le sol environnant le stockage). Nous sommes partis du jeu d'équations réduit suivant :

$$
\begin{aligned}
\frac{\partial T^{*}}{\partial t} & =L^{*} T^{*} \\
L^{*} & =\frac{\lambda^{*}}{(\rho C)^{*}}\left[\frac{\partial^{2}}{\partial x_{1}^{2}}+\frac{\partial^{2}}{\partial x_{2}^{2}}+\frac{\partial^{2}}{\partial x_{3}^{2}}\right] \\
T^{*} & =\frac{\varepsilon \rho_{\mathrm{f}} C_{\mathrm{f}} T_{\mathrm{f}}+(1-\varepsilon) \rho_{\mathrm{c}} C_{\mathrm{c}} T_{\mathrm{c}}}{\varepsilon \rho_{\mathrm{f}} C_{\mathrm{f}}+(1-\varepsilon) \rho_{\mathrm{c}} C_{\mathrm{c}}} \\
(\rho C)^{*} & =\varepsilon \rho_{\mathrm{f}} C_{\mathrm{f}}+(1-\varepsilon) \rho_{\mathrm{c}} C_{\mathrm{c}} .
\end{aligned}
$$

L'indice * caractérise le milieu homogène équivalent au milieu composite air/cailloux :

- $T^{*}$ est la température équivalente du milieu composite ;

- $\lambda^{*}$ est la conductivité équivalente du milieu.

Dans le cas de la relaxation, on peut prendre :

$$
T_{\mathrm{f}}=T_{\mathrm{c}} \text {. }
$$

Conditions initiales :

$$
T^{*}\left(0, x_{1}, x_{2}, x_{3}\right)=T_{\exp }^{\circ}\left(0, \bar{x}_{1}, x_{2}, x_{3}\right) .
$$

$T_{\text {exp }}^{\circ}$ est la température expérimentale au début de la période de modélisation.

Conditions aux limites:

$$
-\lambda * \frac{\partial T^{*}}{\partial \mathbf{n}}=h_{\mathrm{p}}\left(T^{*}-T_{\mathrm{ext}}\right)
$$

$h_{\mathrm{p}}$ dépend de l'isolation thermique et varie donc suivant la face du stockage ; $T_{\text {ext }}$ est la température du sol, considérée comme fixe, ou la température de l'air ambiant pour la face supérieure; $\mathbf{n}$ est la normale à la surface-enveloppe dirigée vers l'intérieur du stockage.

Nous avons donc à résoudre l'équation de la conduction dans un domaine parallélépipédique correspondant à l'enveloppe du stockage ; nous devons adopter un maillage régulier correspondant à celui des sondes thermiques que nous avons présentées au paragraphe 3 ; quant aux conditions aux limites, nous les supposons homogènes sur chaque face du parallélépipède. En fonction de ces données, une méthode par différences finies nous semble la plus adéquate. Nous avons choisi le schéma implicite à directions alternées (A.D.I.) développé par D'Yakonov [15]. Plus précisément, nous avons résolu le système suivant :

$$
\begin{aligned}
& \left(1-\alpha \delta^{2} x_{1}\right) U^{n+1^{*}}\left(x_{1}, x_{2}, x_{3}\right)= \\
& =\left(1+\alpha \delta^{2} x_{2}\right)\left(1+\alpha \delta^{2} x_{3}\right) T^{n}\left(x_{1}, x_{2}, x_{3}\right) \\
& \left(1-\alpha \delta^{2} x_{2}\right) U^{n+1^{* *}}\left(x_{1}, x_{2}, x_{3}\right)= \\
& =U^{n+1^{*}}\left(x_{1}, x_{2}, x_{3}\right)-\left(1+\alpha \delta^{2} x_{3}\right) T^{n}\left(x_{1}, x_{2}, x_{3}\right)
\end{aligned}
$$

$$
\begin{aligned}
& \left(1-\alpha \delta^{2} x_{3}\right) T^{n+1}\left(x_{1}, x_{2}, x_{3}\right)= \\
& =2 U^{n+1^{* *}}\left(x_{1}, x_{2}, x_{3}\right)+\left(1+\alpha \delta^{2} x_{3}\right) T^{n}\left(x_{1}, x_{2}, x_{3}\right)
\end{aligned}
$$

où :

- $T^{n}\left(x_{1}, x_{2}, x_{3}\right)$ est la température au temps $n$, au point $m\left(x_{1}, x_{2}, x_{3}\right)$ du maillage ;

- $\alpha=1 / 2\left(k / h^{2}\right) \kappa(k$ : pas de temps, $l$ : pas d'espace) ;

- $\kappa=\lambda^{*} /(\rho C)^{*}$.

On a :

où :

$$
\begin{aligned}
\delta_{x}^{2} T_{m}^{n} & =T_{m+1}^{n}-2 T_{m}^{n}+T_{m-1}^{n} \\
T_{m}^{n} & =T(x=m l, y, z, t=n k)
\end{aligned}
$$

$U^{n^{*}}$ et $U^{n^{* *}}$ sont des valeurs intermédiaires sans contenu physique précis, utiles pour le calcul. On déduit de la formule précédente les valeurs intermédiaires sur les frontières :

$$
\begin{aligned}
U^{n+1^{*}}= & 1 / 2\left(1-\alpha \delta^{2} x_{2}\right)\left(1-\alpha \delta^{2} x_{3}\right) g^{n+1}+ \\
& +1 / 2\left(1+\alpha \delta^{2} x_{2}\right)\left(1+\alpha \delta^{2} x_{3}\right) g^{n} \\
U^{n+1^{* *}}= & 1 / 2\left(1-\alpha \delta^{2} x_{3}\right) g^{n+1}- \\
& -1 / 2\left(1+\alpha \delta^{2} x_{3}\right) g^{n}
\end{aligned}
$$

$g^{n}$ est la valeur de la température au temps $n$ sur la frontière considérée (les termes d'ordre supérieur en les différences centrées $\delta$ ont été négligés).

Cette écriture demande une résolution en trois étapes, chaque étape impliquant la résolution d'un système tridiagonal le long de lignes parallèles à 
l'axe $x_{1}$ pour la première étape, à l'axe $x_{2}$ pour la seconde, et $x_{3}$ pour la troisième, ce qui est particulièrement performant du point de vue du calcul informatique. La formulation (22) est inconditionnellement stable.

L'enveloppe de béton de $15 \mathrm{~cm}$ d'épaisseur, intérieure à l'isolation, représente une capacité calorifique égale à $1 / 3$ de celle du stockage air/cailloux proprement dit et a une conductivité thermique 3 fois supérieure à celle du milieu air-cailloux. Nous ne pouvions, pour des raisons informatiques, modéliser séparément les deux milieux avec leurs caractéristiques physiques spécifiques. Une étude approfondie [11] a montré que l'on pouvait remplacer ce volume de béton par un volume/enveloppe, composé de matériau composite air/cailloux, et ayant la même capacité calorifique que l'enveloppe de béton ; toutefois, l'on doit modifier les conditions aux limites de façon à tenir compte de la plus forte conductivité du béton.

5.2 COMPARAISON ENTRE DONNÉES ET PRÉDICTIONS DU MODÈLE. - Les figures $6 \mathrm{a}, 6 \mathrm{~b}, 6 \mathrm{c}$ donnent l'évolution des températures mesurées dans le stockage (courbes en pointillés) et les prédictions du modèle (courbes en traits pleins) pour les axes "Haut Couloir», " Milieu », et « Bas Couloir », tous les cinq jours ; les valeurs expérimentales aussi bien que les prédictions du modèle ont été lissées.

Nous avons initialisé le modèle (Eq. (20)) en imposant aux nœuds du maillage coïncidant avec les emplacements des sondes, les températures expérimentales de début de relaxation (courbes supérieures pointillées); pour les autres nœuds, nous avons appliqué une moyenne pondérée. Ici aussi l'absence de sondes, notamment près des parois de béton, a posé quelques problèmes et nous ne pouvons affirmer que modèle et expérience partent exactement des mêmes valeurs de températures. Pour la température extérieure (sol et air), nous nous sommes basés sur les données mensuelles de température d'air et de température de sol à différentes profondeurs fournies par la météorologie nationale pour les stations de Nice et Montélimar et nous avons évalué la température à $2,5 \mathrm{~m}$ de profondeur et le réchauffement du sol au cours du temps dû aux pertes du stockage. Nous avons pris les températures suivantes :

Date Température d'air Température de sol

$\begin{array}{rll}\overline{9} & -\mathrm{C} & -\mathrm{C} \\ 18 / 4 & 13^{\circ} \mathrm{C} & 1{ }^{\circ} \mathrm{C} \\ 23 / 4 & 15^{\circ} \mathrm{C} & 20^{\circ} \mathrm{C} \\ & 17,5^{\circ} \mathrm{C} & 21^{\circ} \mathrm{C}\end{array}$

Le pont thermique par la dalle inférieure nous a conduit à augmenter le coefficient de pertes correspondant $h_{\text {inf }}=0,32 \mathrm{~W} / \mathrm{m}^{2} .{ }^{\circ} \mathrm{C}$.

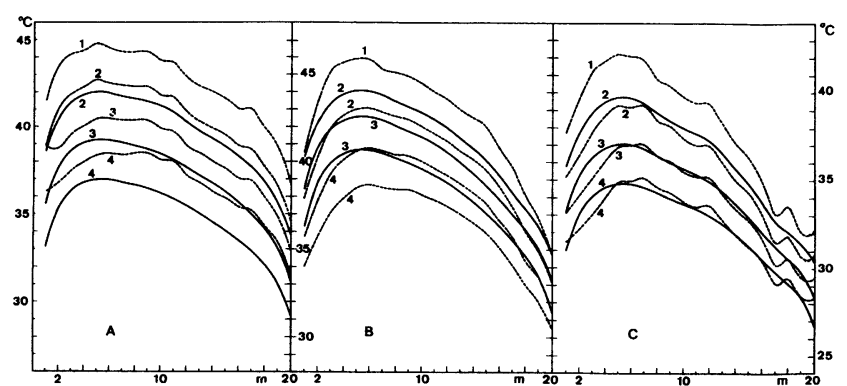

Fig. 6. - Comparaison des données expérimentales (lignes pointillées) et des prédictions du modèle (lignes continues) pour une relaxation du stockage initialement chargé $\left(\lambda_{\text {eq }}=0,45 \mathrm{~W} / \mathrm{m} .{ }^{\circ} \mathrm{C}\right)$. Les courbes (1) indiquent les températures initiales ; les courbes (2), (3), (4) correspondent aux cartes de températures après 6,13 et 18 jours de relaxation.

Courbes A : températures le long de l'axe supérieur (côté galerie technique);

Courbes B : températures le long de l'axe central ;

Courbes $\mathrm{C}$ : températures le long de l'axe inférieur (côté mur).

L'abscisse est la distance à l'entrée du stockage.

[Comparison between experiments data (dotted curves) and model predictions (full curves) for an initially charged thermal storage $\left(\lambda_{\mathrm{eq}}=0.45 \mathrm{~W} / \mathrm{m} .{ }^{\circ} \mathrm{C}\right)$. Curves (1) indicate initial temperatures. Curves (2), (3), (4) indicate temperatures after respectively $6,13,18$ days of relaxation working rate.

Curve A : temperatures along the top axis (technical gallery side).

Curve B : temperatures along the central axis.

Curve $\mathrm{C}$ : temperatures along the bottom axis (wall side). Temperatures are plotted against distance from the entry surface of the storage (pebbles).]

Prendre une température de terre uniforme est certainement une approximation grossière. Quant à la température d'air adoptée, elle ne permet pas de tenir compte du réchauffement de la dalle supérieure pendant la journée, ni des pertes par rayonnement nocturne à grandes longueurs d'onde. Néanmoins ces approximations sont raisonnables au vu de la durée des cycles observés. La conductivité équivalente $\lambda^{*}$ a été prise égale à $0,45 \mathrm{~W} / \mathrm{m} .{ }^{\circ} \mathrm{C}$.

$\mathrm{Au}$ vu des figures (6), l'importance des courants convectifs dans le stockage apparaît clairement. L'analyse des données expérimentales (paragraphe 4) a montré que la convection naturelle s'atténuait au bout d'une quinzaine de jours.

Les figures 7A, B, C comparent valeurs expérimentales et prédictions du modèle lorsqu'on initialise le modèle avec les données expérimentales observées au bout de neuf jours de relaxation : l'écart entre températures expérimentales et températures données par le modèle est moindre. Néanmoins, les pertes par la dalle supérieure et vers les plenums 


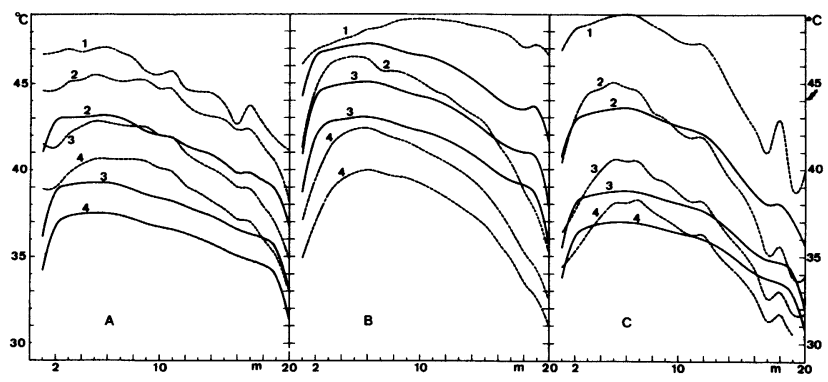

Fig. 7. - Même réseau de courbes qu'en figure 6. Les courbes $1,2,3,4$ correspondent respectivement à des relevés après 9 jours, 14 jours, 19 jours et 24 jours de relaxation thermique.

[Some-curves as on figure 6. Curves (1), (2), (3), (4) are obtained after, respectively, 9, 14,19 and 24 days of thermal relaxation.]

d'entrée et de sortie entretiennent de faibles courants convectifs [20,27].

Dans la référence [10], nous comparons données expérimentales et prédictions du modèle dans le cas d'une relaxation à partir d'un stockage partiellement plein. Même en initialisant la simulation à 10 jours de relaxation, les valeurs expérimentales et simulées restent relativement éloignées car des courants convectifs apparaissent entre la partie chaude et la partie froide du stockage dont on a vu les effets sur l'avancée du front (Fig. 5).

\section{Cycle complexe proche des conditions réelles.}

Après des expériences d'assez courte durée s'intéressant à un mode particulier de fonctionnement (stockage, déstockage, ou relaxation), une étude combinant les différents modes a été entreprise afin d'estimer les performances d'un tel système lors d'un

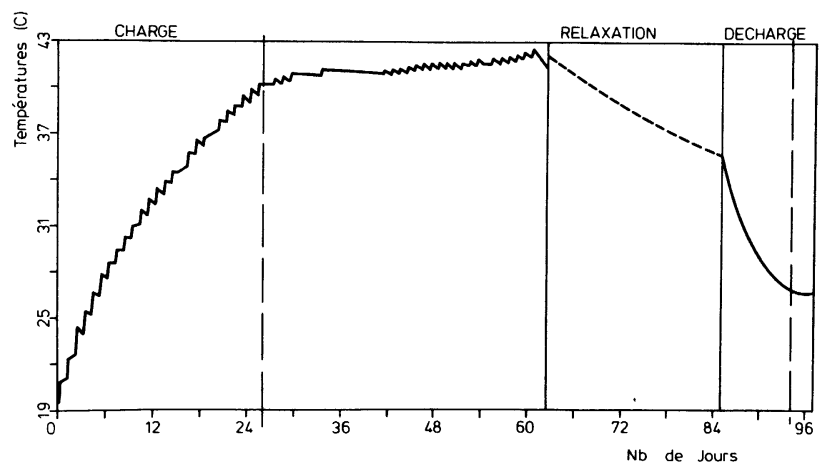

Fig. 8. - Evolution de la température moyenne du stockage lors d'un cycle complexe proche des conditions réelles (charge, relaxation, puis décharge).

[Mean thermal storage temperature evolution for a complex, close to reality, cycle (charge, relaxation, discharge working rates).] fonctionnement réel sur une longue période : « cycle long terme ». Ce cycle comporte 3 phases :

- une charge pendant 62 jours : air insufflé à 50$60^{\circ} \mathrm{C}$, à un débit moyen de $1500 \mathrm{~m}^{3} / \mathrm{h}, 6 \mathrm{~h}$ par jour ;

- une relaxation pendant 22 jours ;

- une décharge pendant 12 jours : air extérieur insufflé en continu, à débit moyen de $880 \mathrm{~m}^{3} / \mathrm{h}$.

L'évolution de la température moyenne du stockage est représentée en figure 8 . On la complète sur les figures 9 et 10, par les fluctuations de débit, de températures d'entrée et de sortie d'air, pour les phases de charge et décharge respectivement. Charge et décharge ont été arrêtées au bout de, respectivement, 2 mois et 12 jours, compte tenu de la stabilisation des niveaux correspondants. De même, il a été décidé d'arrêter la relaxation au bout de 22 jours, au vu de la dégradation du niveau thermique.

Le bilan énergétique est résumé sur le tableau IV. A l'aide de ces résultats, on se propose d'évaluer l'intérêt de ce type de stockage.

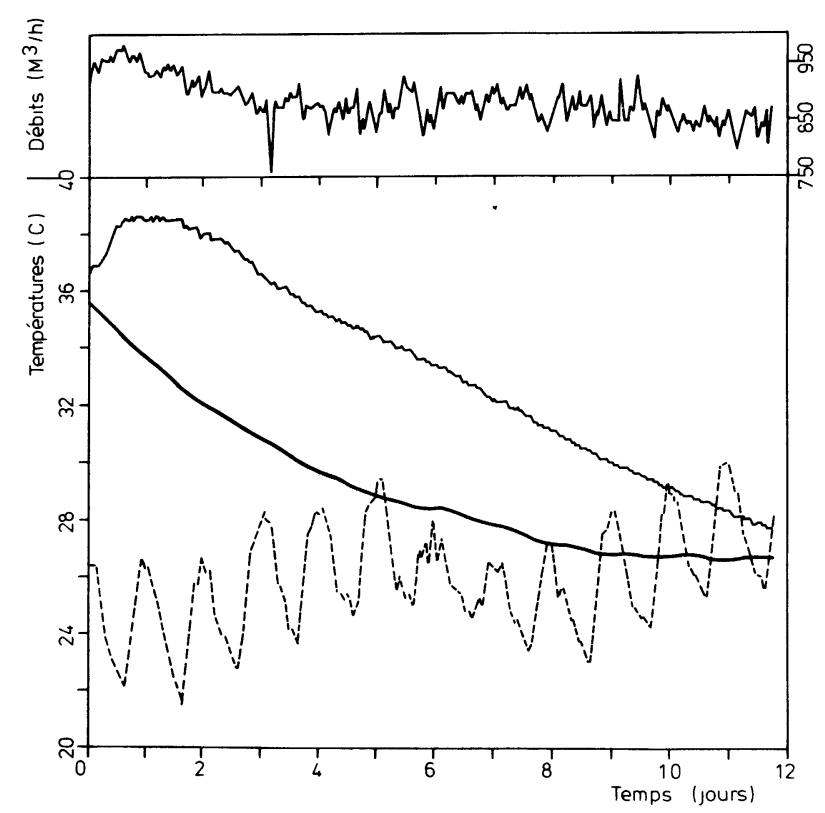

Fig. 9. - Evolution du stockage lors d'un cycle de charge thermique proche des conditions réelles (températures variables selon les conditions climatiques ; charge thermique diurne).

$\square$ : débit d'air à l'entrée du stockage ;

+ : température d'air à l'entrée ;

- : température moyenne du stockage ;

* : température de l'air extrait du stockage.

[Storage evolution during a realistic thermal charge cycle (the insufflated air temperature depends on climatic conditions); diurnal thermal charge only.

$\square$ : flow rate at the entry of storage ;

$+\quad$ : air temperature at the entry;

- : mean temperature of the storage ;

* : extracted air temperature.] 


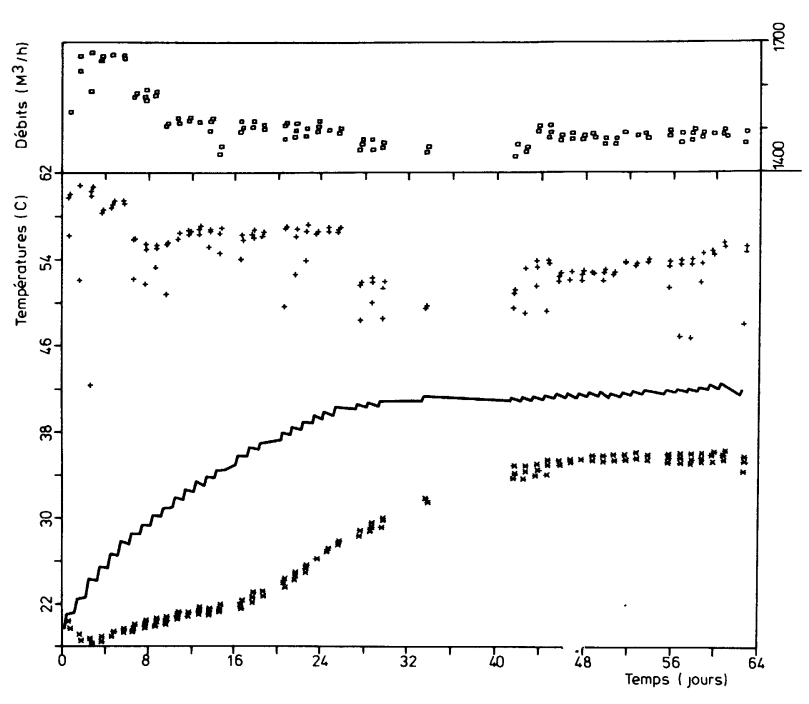

Fig. 10. - Evolution du stockage au cours d'un cycle de décharge proche des conditions réelles de fonctionnement. A : débit de l'air en entrée du stockage ; B : température de l'air extrait ; $\mathrm{C}$ : température moyenne du stockage ; D : température de l'air soufflé en entrée du stockage.

[Storage evolution during a realistic thermal discharge cycle. A : flow rate at the entry; $\mathrm{B}$ : extracted air temperature ; $\mathrm{C}$ : mean temperature of the storage ; $\mathrm{D}$ : insufflated air temperature.]

Rendements. - On définit le rendement global $\eta_{\mathrm{G}}$ de l'unité de stockage comme le rapport de la quantité d'énergie récupérée à la quantité d'énergie fournie. Pour ce cycle: $\eta_{\mathrm{G}}=0,14$. Ce rendement est faible. Cependant, on peut faire les remarques suivantes :

a) $90 \%$ de la charge est obtenue au bout de 26 jours ; on ne l'accroît que de $10 \%$ au cours des 36 jours suivants. Ceci s'explique d'une part par des températures d'air insufflé assez élevées au début $\left(60^{\circ}\right)$, plus faibles ensuite $\left(48\right.$ à $\left.55^{\circ} \mathrm{C}\right)$, d'autre part par l'augmentation des pertes thermiques corrélativement à l'augmentation du niveau thermique de l'unité.

b) En pratique, par un choix adéquat de capteurs à air, et une isolation poussée des gaines de distribution, on peut espérer insuffler de l'air à $65^{\circ} \mathrm{C}$, voire $70{ }^{\circ} \mathrm{C}$.

c) $95 \%$ de la décharge est obtenue au bout de 9 jours. En effet, pour des raisons de calendrier, la décharge s'est effectuée en juillet, en insufflant l'air extérieur dont la température moyenne était de $25,8^{\circ} \mathrm{C}$ (Fig. 10). En pratique l'air insufflé dans le stockage proviendrait de l'habitat l'hiver et aurait une température voisine de $18{ }^{\circ} \mathrm{C}$.

Pendant toute la durée des phases de charge et décharge, les gaines d'accès aux plenums sont restées ouvertes. En fermant ces gaines pendant les $18 \mathrm{~h}$ de non-charge, et pendant la phase de relaxation, on peut éliminer une grande partie des fuites aérauliques.

Ainsi, à titre indicatif, avec un rendement de

Tableau IV. - Bilan énergétique (en $\mathrm{kWh}$ ) du cycle complexe de longue durée (charge, relaxation, décharge) représenté en figure 8. Lénergie stockée est affectée du signe + , l'énergie perdue ou extraite du signe -. Le cycle de charge se décompose en deux périodes : charge à $90 \%$ (0-26 jours), puis charge des $10 \%$ résiduels (27-62 jours); de même le cycle de décharge peut être décomposé en une partie où l'on récupère $95 \%$ de l'énergie et une partie où l'on récupère, difficilement, les $5 \%$ résiduels.

[Energy balance (in $\mathrm{kWh}$ ) for a long term, complex, cycle (charge, relaxation, discharge), presented in figure 8. The energy stored is indicated by mark (+), the energy lost or extracted, by mark $(-)$. The charge cycle is splitted in two periods : storage filled at $90 \%$ (days 0 to 26); storage of the residual $10 \%$ (days 27 to 62 ); the discharge cycle may also be splitted in two parts : the first one where one recovers $95 \%$ of the available energy and a second one where one recovers, not easily, the remaining $5 \%$.]

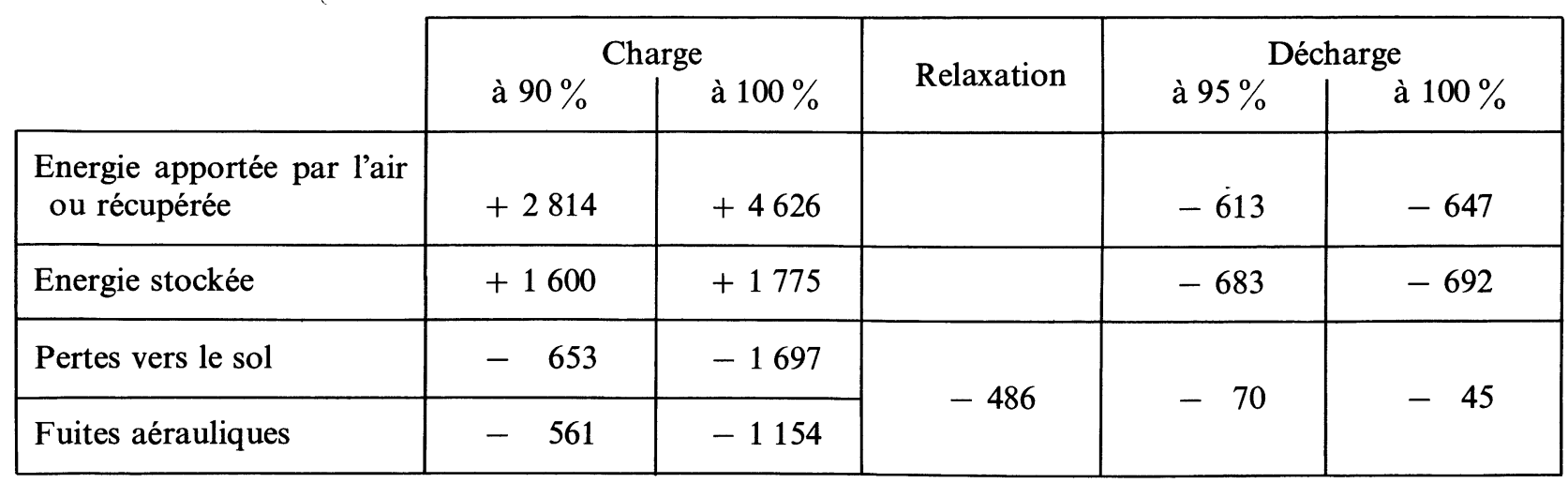


décharge de 0,9 , on pourrait atteindre un rendement global de :

$$
\eta_{\mathrm{G}}=\frac{\text { Energie récupérée }}{\text { Energie fournie à } 90 \%}=0,46 \text {. }
$$

Il apparait clairement que la phase de relaxation doit être réduite au minimum. Il ne s'agit donc pas d'un stockage intersaisonnier mais plutôt d'un stockage de quelques semaines.

\section{Conclusions.}

Dans cette étude, nous avons vérifié les avantages, $a$ priori, d'un stockage en lit de galets ventile :

- bonne stratification thermique à la charge et à la décharge ;

- bonne répartition aéraulique dans la masse caillouteuse à condition de s'assurer une granulométrie homogène. A débit faible, il est préférable de prévoir, en mode charge, l'arrivée de l'air en partie basse du réservoir, la reprise pouvant se faire en partie haute.

Par contre, nous avons mis en évidence un certain nombre de problèmes qui peuvent détruire l'efficacité d'un tel stockage :

- convection naturelle qui, pendant les périodes de relaxation, dans le cas d'un stockage horizontal partiellement chargé, détériore partiellement la stratification, et dans le cas d'un stockage plein peut augmenter les pertes thermiques;

- pertes aérauliques qu'il convient de limiter au maximum en apportant un soin particulier à l'étanchéité à la construction, et à l'aide de systèmes automatiques de fermeture des volets d'étanchéité vers les gaines d'accès ;

- pertes thermiques qui, même si l'isolation du réservoir est bonne, vont avoir des effets non négligeables sur les longues périodes de fonctionnement. Le sol joue un rôle important: certains stockages de très gros volumes incluent la récupération de la chaleur dans le sol environnant.

La modélisation de l'évolution de l'état d'un stockage en galets ventilé, lors des différentes phases . de fonctionnement est complexe ; ceci principalement à cause de l'imbrication de phénomènes relevant de la mécanique des fluides et de la thermique : on se trouve devant un milieu divisé proche d'un milieu poreux. Nous avons modélisé la conduction dans le milieu composite et dans l'enveloppe à l'aide d'une méthode aux différences finies implicite à direction alternée, solution qui semble assez bien adaptée à ce type de problème à maillage régulier avec pertes homogènes pour chaque face. Néanmoins, les effets conducto-convectifs étant prépondérants, pendant les périodes de relaxation notam- ment, nous pensons qu'une modélisation fine de ces phénomènes serait nécessaire.

En ce qui concerne le coefficient de transfert thermique air/cailloux, nous avons vu que dans toutes les formulations, il est donné en fonction d'une vitesse moyenne considérée comme uniforme dans le lit. Tous les effets d'irrégularités de l'écoulement dûs aux diverses inhomogénéités du lit ainsi qu'au type de surface et à la forme des particules solides sont incluses dans un coefficient numérique expérimental. Une étude expérimentale paramétrique plus complète et plus soignée du coefficient de transfert thermique dans le milieu air/cailloux serait nécessaire.

La conductivité équivalente du milieu air/cailloux est un paramètre important, difficile à déterminer avec précision dans ce milieu composite proche d'un milieu poreux. Nos conditions expérimentales complexes ne nous ont permis qu'une détermination grossière de ce coefficient [11]. Seule une expérience de laboratoire très soignée pourrait en donner une valeur précise.

A notre connaissance, l'expérience de Sophia Antipolis est la première étude expérimentale d'un stockage thermique en lit de cailloux ventilé de volume important. Elle a déjà mis en évidence un fait significatif : la technique mise en cuvre ici ne permet pas d'avoir de bons rendements de déstockage au-delà d'un mois d'utilisation.

La conception même du prototype, essentiellement tourné vers l'évaluation des performances thermique et aéraulique, ne nous a pas permis d'étendre l'étude sur le plan de l'analyse économique. Il serait pourtant intéressant de comparer les qualités thermiques mais aussi la rentabilité économique de ce type de stockage à celles d'autres types de système de grande capacité actuellement à l'étude, en particulier dans les pays de l'Europe du Nord et au Canada. Les premières années des suivis expérimentaux [28-30] semblent mettre l'accent sur la complexité mais aussi la nécessité d'une approche système de tels projets ; la recherche d'un optimum économique demande un pilotage délicat où le nombre de paramètres à prendre en compte et par cela même le nombre de modes de fonctionnement possibles est important.

\section{Remerciements.}

Cette expérience et les études qui s'y rattachent ont été en large partie financées par l'Agence Française pour la Maîtrise de l'Energie (A.F.M.E.) qui a pris le relais du COMES en 1982, et que nous tenons à remercier. Ce travail a également bénéficié du soutien de la Direction du C.S.T.B. et de la Direction du PIRSEM/CNRS ; nous les remercions pour leur soutien constant depuis le début de cette étude. Nous remercions également les membres de 
l'Etablissement C.S.T.B. de Sophia Antipolis et de l'Equipe RAMSES qui ont contribué depuis 1981 au bon déroulement de cette collaboration scientifique entre les deux équipes.

\section{Bibliographie}

[1] Alanis, E., Saravia, L. and Rovetta, L., Measurement of rock pile heat transfer coefficient, Sol. Energy 19 (1977) 571-572.

[2] Boisdet, A., Peube, J. L. et Blay, D., Stockage thermique en milieu poreux granulaire, Revue Phys. Appl. 17 (1982) 591-594.

[3] Bourdeau, L., Delcambre, B., Martin, J. et RuBINSTEIN, M., Stockage thermique de longue durée par lit de cailloux, Cah. Cent. Sci. Tech. Bâtim., n 1856 (Editions du C.S.T.B., rue du Recteur-Poincaré, 75016 Paris), 1983.

[3 bis] BourdeAu, L., Stockage intersaisonnier d'énergie solaire sur lit de cailloux enterrés, Rev. Gén. Therm. $22 \mathrm{n}^{\circ} 254$ (1983) 125-129.

[4] Chandra, P. and Willits, D. H., Pressure drop and heat transfer characteristics of air-rock bed thermal storage systems, Sol. Energy 27 (1981) 547-554.

[5] Clement, D., Etude expérimentale des systèmes de chauffage solaire pour habitat individuel avec capteurs solaires à air et stockage thermique dans un réservoir de cailloux, Thèse de Doctorat de $3^{\mathrm{e}}$ Cycle, Université de Paris 7, Paris, octobre 1983 (Bibliothèque de l'Université de Paris 7, 2, place Jussieu, 75251 Paris Cedex 05) 1983.

[6] Close, D. J., Rock pile thermal storage for comfort air conditioning, Mech. Chem. Eng. Trans. MC 1 (1965) 11-22.

[7] Coutier, J. and FARber, E. A., Two applications of a numerical approach heat transfer process within rock beds, Sol. Energy 29 (1982) 451-462.

[8] Courrege, Ph. et DANG Vu, Cl., Le stockage à long terme de la chaleur en lit de roches, Publication interne. Equipe RAMSES (E.R. 293 du CNRS), Bât. 508, Université, 91405 Orsay (1979).

[9] DANG Vu, Cl. and RIVET, P., Interseasonal thermal storage in a large rock-bed connected to air solar collectors (numerical simulation), Proceedings of the International Conference on Building Energy Management (I.C.B.E.M.) held at Povoa de Varzim (Portugal), May 1980 (Pergamon Press, Oxford) 1980 , p. 499-506.

[10] DANG VU, Cl. and MARTIN, J., Experimentation on a long term rock-bed thermal storage, First E.C. Conference on Solar Heating. Proceedings of the International Conference held at Amsterdam, April 30-May 4, 1984 (D. Reidel publishing Company) 1984, p. 1016-1020.

[11] Delcarte-Dang Vu, Cl., Etude expérimentale et modélisation du stockage thermique de longue durée en lit de cailloux enterré, ventilé. Thèse de Doctorat en Sciences de l'Université Paris 7, 28 juin 1985 (Bibliothèque de l'Université Paris 7, 2, place Jussieu, 75251 Paris Cedex 05) 1985.
[12] Denton, W. H., The heat transfer and flow resistance for fluid through randomly packed spheres. In general discussion on heat transfer. Inst. Mech. Engrs. and A.S.M.E., London (1951), 370-373.

[13] Donnadieu, G., Transmission de la chaleur dans les milieux granulaires. Etude du lit fixe et du lit fluidisé, Thèse d'Ingénieur Docteur, Université de Paris, Rev. Inst. Fr. Pét., Ann. Combust. Liq. (1960).

[14] Dunkle, R. V. and Ellul, W. M., Randomly packed particulate bed regenerators and evaporative coolers, Mech. Eng. Trans. MC 8 (1972) 117-121.

[15] D'Yakonov, Ye. G., On the application of disintegrating difference operators, Zh. Vycisl. Mat. Mat. Fiz 3 (1963) 385-388.

[16] Furnas, C. C., Heat transfer from a gas stream to a bed broken solids, Trans. Am. Inst. Chem. Eng., Detroit Meeting 29 (1930) 174-193.

[17] Guidoune, H., Lavaine, F. et Point N., Résolution théorique et numérique d'un modèle de déstockage de l'énergie solaire. Méthodes numériques pour les sciences de l'ingénieur, $2^{\mathrm{e}}$ Congrès GAMNI, 1980 (Dunod) 1981.

[18] Handley, D. and Heggs, P. J., Momentum and heat transfer mechanisms in regular shaped packings, Trans. Inst. Chem. Eng. 46 (1968) 251264.

[19] Horton, C. W. and Rogers, F. T. Jr., Convection currents in a porous medium. J. Appl. Phys. 16 (1945) 367-370.

[20] Joubert, P., Convection naturelle instationnaire en milieu confiné stratifié, Thèse de Doctorat de $3^{\text {e }}$ Cycle de l'Université de Poitiers, mars 1985 (Bibliothèque de l'Université, 40, avenue du Recteur-Pineau, 86000 Poitiers) 1985.

[21] LAPWOOD, E. R., Convection of a fluid in a porous medium, Proc. Cambridge Philos. Soc. 44 (1948) 508-521.

[22] LöF, G. O. G. and HAWLEY, R. W., Unsteady state heat transfer between air and loose solids, Ind. Eng. Chem. 40 (1948) 1061-1070.

[23] Mandal, S. K. and Maheshwari, B. L., Forced convective heat transfer in a bed or rock pile, I.E. (I) ME (1972) 98-104.

[24] Saez, A. E. and McCoy, B. J., Transient analysis of packed bed thermal storage systems, Int. J. Heat Mass Transfer 26 (1983) 49-54.

[25] SAunder, O. A. and Ford, H. J., Heat transfer in the flow of gas through a bed of solid particles, $J$. Iron Steel Inst. CXLI (1940) 291.

[26] SchumanN, T. E. W., Heat transfer : a liquid flowing through a porous prism, J. Franklin Inst. 208 (1929) 405-416. 
[27] VisKanTA, R. and KIM, D. M., Effect of a wall heat conduction on natural convection heat transfer in a square enclosure, J. Heat Transfer 107 (1985) 139-146.

Conférences, cours, bulletins.

[28] ENERSTOCK-83, 2nd International Conference on Subsurface Heat Storage in Theory and Practice, Proceedings-Swedish Council for Building Research, Stockholm, June 6-8, 1983.

[29] ENERSTOCK-85, 3rd International Conference on Energy Storage for Building Heating and Coo- ling, Proceedings, Toronto, September 22-26, 1985.

[30] «Stockage Thermique. Nouvelles Internationales». Bulletin $n^{\circ} 1$ à 6 . Bulletin périodique d'informations, édité par "SIGMA-Consultants" avec le soutien de l'A.F.M.E., Sophia Antipolis/Valbonne (Y. Boileau).

[31] Löf, G. O. G., Solar Air Systems, Solar Energy Conversion, an introductory course, A. E. Dixon, J. D. Leslie Editors (Pergamon Press) 1978, p. 331-375. 